\title{
VALIDAÇÃO DE METODOLOGIA ANALÍTICA PARA DOSEAMENTO DE SOLUÇÕES DE LAPACHOL POR
} CLAE

\section{Said G. C. Fonseca}

Departamento de Farmácia, Faculdade de Farmácia, Odontologia e Enfermagem, Universidade Federal do Ceará,

Rua Capitão Francisco Pedro, 1210, 60430-370 Fortaleza - CE

Leila B. L. da Silva, Rebeka F. Castro e Davi P. de Santana*

Departamento de Farmácia, Faculdade de Farmácia, Universidade Federal de Pernambuco, Rua Prof. Arthur de Sá, s/n, Cidade Universitária, 50750-520 Recife - PE

\begin{abstract}
VALIDATION OF THE ANALYTICAL METHODOLOGY FOR EVALUATION OF LAPACHOL IN SOLUTION BY HPLC. Lapachol is a naphthoquinone found in several species of the Bignoniaceae family possessing mainly anticancer activity. The present work consists of the development and validation of analytical methodology for lapachol and its preparations. The results here obtained show that lapachol has a low quantification limit, that the analytical methodology is accurate, reproducible, robust and linear over the concentration range $0.5-100 \mu \mathrm{g} / \mathrm{mL}$ of lapachol.
\end{abstract}

Keywords: lapachol; validation; HPLC.

\section{INTRODUÇÃO}

O lapachol [2-hidroxi-3-(3-metil-butenil)-1,4-naftoquinona] (1) é uma naftoquinona encontrada em diversas espécies vegetais da família Bignoniaceae, especialmente aquelas do gênero Tabebuia. Possui atividade antimicrobiana contra bactérias e fungos, antiviral, antiinflamatória e principalmente anticâncer, atuando sobretudo nos tumores sólidos ${ }^{1}$.

Em virtude de suas propriedades farmacológicas, este ativo tem sido bastante estudado visando sua utilização na terapêutica. Como passo inicial de um estudo de desenvolvimento galênico com a dro$\mathrm{ga}^{2}$, faz-se necessário o desenvolvimento e A validação de uma metodologia analítica capaz de quantificar a droga e os produtos farmacêuticos preparados a partir dela.

Os objetivos deste trabalho consistem no desenvolvimento e validação de metodologia analítica para o doseamento do lapachol e suas preparações.

\section{MATERIAIS E MÉTODOS}

\section{Materiais e equipamentos}

Lapachol Sigma, Lote: 33H34575; $\beta$-lapachona preparado pelo Instituto de Antibióticos da UFPE; metanol grau UV/HPLC; água purificada obtida através de Milli-Q para uso em CLAE. Os demais reagentes e solventes utilizados foram de grau analítico. Sistema de cromatografia líquida de alta eficiência HP-Série 1100 (Hewlet Packard), munido de degaseificador, bomba quaternária, injetor manual com loop de $20 \mu \mathrm{L}$, coluna cromatográfica Lichrosorb RP-18 250x4 mm (Merck), detector UV e integrador HP 3395.

\section{Metodologia}

A escolha da fase móvel foi realizada através da mistura binária de solventes constituídos por metanol e solução aquosa de ácido

*e-mail: d-santana@bol.com.br<smiles>CC(C)=CCC1=C(O)C(=O)c2ccccc2C1=O</smiles>

(1)<smiles>CC1(C)CCC2=C(O1)c1ccccc1C(=O)C2=O</smiles>

acético a 5\%, partindo-se da proporção 95:5 até 70:30, observando o cromatograma quanto ao perfil cromatográfico e resolução entre o pico do lapachol (1) e da $\beta$-lapachona (2), fixando-se o fluxo em $1 \mathrm{~mL} / \mathrm{min}$. Foi selecionado, em termos de sensibilidade e repetibilidade, o melhor comprimento de onda a ser utilizado na detecção do lapachol, dentre os comprimentos de 251, 278 e $331 \mathrm{~nm}$ citados na literatura ${ }^{3}$.

A validação da metodologia analítica foi determinada segundo os critérios descritos na norma ICH "International Conference on Harmonization of Thechnical Requirements for Registration of Pharmaceuticals for human Use" tópico Q-2b, de $1996^{4}$. Almejando as melhores e mais reprodutíveis condições de integração, as concentrações de $1,2,5$, e $8 \mu \mathrm{g} / \mathrm{mL}$ foram repetidamente injetadas e cada cromatograma obtido foi reintegrado usando o parâmetro Ruído ("Threshold") em diferentes valores, sendo possível a construção de 9 curvas de calibração e seus respectivos coeficientes linear e angular da reta, os quais foram comparados. Os valores de área de uma das concentrações $(1 \mu \mathrm{g} / \mathrm{mL})$ também foram diretamente comparados entre diferentes pares de valores de ruído em relação àquele considerado como mais adequado para a construção da curva de calibração, através da análise de variância entre duas médias, objetivando constatar a existência ou não de significância na sua diferença. Cada cromatograma teve a altura do pico de lapachol medida com paquímetro $( \pm 0,002$ $\mathrm{cm}$ ), sendo os valores encontrados corrigidos para seu equivalente em atenuação zero $\left(\mathrm{ATT}^{\wedge} 0\right)$, segundo a especificação do fabricante do integrador, e todos os ensaios acima descritos foram repetidos usando os valores de altura dos picos em lugar da área.

Para avaliação da repetibilidade, uma mesma concentração foi repetidamente injetada pelo mesmo operador e os valores de área, 
tempo de retenção, largura e altura dos picos obtidos foram registrados e comparados.

A reprodutibilidade, em termos de precisão intermediária, foi avaliada através da comparação entre as injeções de uma mesma concentração realizada em dias diferentes e por analistas diferentes, inferindo-se os resultados através da análise de variância.

A linearidade foi avaliada pela aplicação de soluções constituídas de diluições autênticas de lapachol padrão em metanol nas concentrações de $0,01 \mu \mathrm{g} / \mathrm{mL}$ até $1000 \mu \mathrm{g} / \mathrm{mL}$, que foram injetadas em triplicata no cromatógrafo e registraram-se os valores de área e altura dos picos encontrados. Os valores foram acompanhados até constatar a continuidade ou não da resposta linear entre concentração e área e/ou altura do pico, avaliando-se segundo o modelo linear pelo método dos mínimos quadrados.

Os limites de detecção e de quantificação foram determinados matematicamente através da relação entre o desvio padrão da curva de calibração e sua inclinação, usando o fator multiplicador apropriado conforme sugerido pela norma $\mathrm{ICH}^{4}$.

A robustez do método foi avaliada mediante a utilização de fases móveis ligeiramente diferentes, preparadas através da mistura dos componentes em balão volumétrico, proveta ou realização da mistura no próprio equipamento.

\section{RESULTADOS E DISCUSSÃO}

Trabalhos anteriores ${ }^{5,6}$ relatam a utilização de fase móvel constituída de acetonitrila : solução aquosa de ácido acético a 0,25\% (1:1), fluxo de $2 \mathrm{~mL} / \mathrm{min}$ e coluna RP-18, obtendo-se resolução entre lapachol e quinonas relacionadas, incluindo metabólitos como $\alpha-\mathrm{e}$ $\beta$-lapachona. Em virtude do custo mais elevado da acetonitrila em relação ao metanol, testou-se a substituição daquele solvente como componente da fase móvel, ao mesmo tempo em que se buscava manter a resolução sob um fluxo menor. Foi conseguida, com a mistura constituída de metanol : solução aquosa de ácido acético a 5\% (80:20), fluxo de $1 \mathrm{~mL} / \mathrm{min}$, coluna Hibar Lichrosorb RP-18 $250 \mathrm{x}$ $4 \mathrm{~mm}$, num tempo de corrida de $7 \mathrm{~min}$, uma boa resolução $(4,53)$ entre o Lapachol e a $\beta$-lapachona, como mostrado na Figura 1, onde se demonstra a especificidade do método em relação aos analitos citados. Com relação à seleção do melhor comprimento de onda a ser utilizado para doseamento do lapachol, os resultados (Tabela 1) mostram que, apesar da detecção no comprimento de onda de $251 \mathrm{~nm}$ apresentar maior sensibilidade (demonstrada pela área média maior que nos demais comprimentos), houve maior variabilidade entre os valores, demonstrada pelo desvio padrão da média e pelo respectivo coeficiente de variação, bem como pelos tipos diferentes de integração. Com relação à detecção no comprimento de onda de $331 \mathrm{~nm}$, o valor integrado é cinco vezes menor que aquele obtido a $278 \mathrm{~nm}$, demonstrando pequena sensibilidade de detecção neste comprimento de onda. Em virtude disto, foi selecionado o comprimento de onda de $278 \mathrm{mn}$.

O estudo de linearidade com injeção das concentrações de $0,01 \mu \mathrm{g} / \mathrm{mL}$ até $1000 \mu \mathrm{g} / \mathrm{mL}$, em triplicata, encontra-se na Tabela 2.

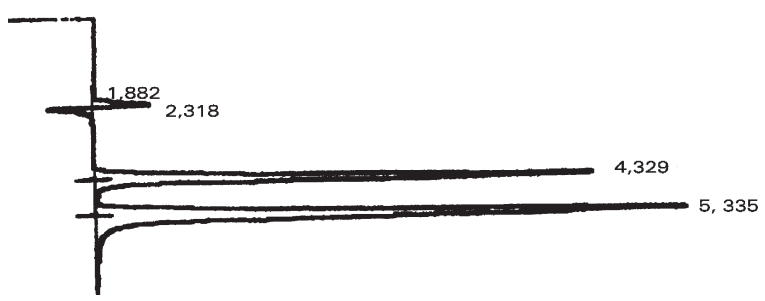

Figura 1. Perfil cromatográfico de $\beta$-Lapachona $($ tr=4,329) e Lapachol $(\operatorname{tr}=5,335)$
Tabela 1. Área dos picos referentes a amostras de lapachol obtidos sob diferentes comprimentos de onda de detecção

\begin{tabular}{lccc}
\hline Injeção e & \multicolumn{3}{c}{ Comprimentos de onda } \\
Resultados & $251 \mathrm{~nm}$ & $278 \mathrm{~nm}$ & $331 \mathrm{~nm}$ \\
\hline 1 & 1.459 .371 & 850.099 & 178.613 \\
2 & 1.383 .330 & 852.727 & O valor muito \\
3 & 1.241 .549 & 832.776 & pequeno da área, \\
4 & 1.205 .572 & 859.724 & comparado com os \\
5 & 1.253 .367 & 857.736 & anteriores, mostrou \\
Média & $1.308 .637,8$ & $850.612,4$ & ser inútil continuar \\
Desvio Padrão & $107.793,5$ & $10.684,6$ & com as leituras \\
C.V. (\%) & $8,237 \%$ & $1,256 \%$ & neste $\lambda$ de onda \\
\hline
\end{tabular}

Tabela 2. Áreas e alturas dos picos obtidos a partir das concentrações de lapachol em solução

\begin{tabular}{|c|c|c|}
\hline \multirow{2}{*}{$\begin{array}{l}\text { Concentração } \\
(\mu \mathrm{g} / \mathrm{mL})\end{array}$} & \multicolumn{2}{|c|}{ Resposta } \\
\hline & Área & Altura $\left(\operatorname{att}^{\wedge} 0\right)(\mathrm{cm})$ \\
\hline \multirow[t]{3}{*}{0,01} & $22.835 \mathrm{PV}$ & - \\
\hline & $17.570 \mathrm{VV}$ & - \\
\hline & $2.761 \mathrm{PV}$ & - \\
\hline \multirow[t]{3}{*}{0,05} & 37.764 PV & 3,623 \\
\hline & 39.069 PV & 3.561 \\
\hline & 37.990 PV & 3.619 \\
\hline \multirow[t]{3}{*}{0,10} & $68.253 \mathrm{PV}$ & 7.302 \\
\hline & 69.747 PV & 7.242 \\
\hline & $72.034 \mathrm{PV}$ & 7.402 \\
\hline \multirow[t]{3}{*}{0,50} & 278.122 PB & 33.736 \\
\hline & 276.757 PB & 33.216 \\
\hline & 276.727 PB & 33.504 \\
\hline \multirow[t]{3}{*}{1,00} & $533.832 \mathrm{~PB}$ & 65.872 \\
\hline & $535.091 \mathrm{~PB}$ & 65.472 \\
\hline & 531.170 PB & 65.472 \\
\hline \multirow[t]{3}{*}{10,00} & 5.466.730 PB & 690.816 \\
\hline & 5.626.221 VB & 707.584 \\
\hline & 5.702.128 VB & 721.280 \\
\hline \multirow[t]{3}{*}{20,00} & 10.662.224 PB & 1352.704 \\
\hline & 10.589.360 PB & 1353.216 \\
\hline & 10.565.024 PB & 1363.456 \\
\hline \multirow[t]{3}{*}{100,00} & 56.901.056 ISPH & 6987.776 \\
\hline & 56.800 .832 ISPH & 6840.320 \\
\hline & 57.015.808 ISPH & 6893.568 \\
\hline 1000,00 & 240.266.880 ISHH & - \\
\hline
\end{tabular}

Foi obtida a linearidade do método analítico na faixa de estudo de 0,05 a $100 \mu \mathrm{g} / \mathrm{mL}$ para dados medidos em altura e de 0,1 a $20 \mu \mathrm{g} / \mathrm{mL}$ para os dados medidos em área.

Injeções de uma mesma concentração $(20 \mu \mathrm{g} / \mathrm{mL})$ realizadas no mesmo dia e mesmo laboratório, pelo mesmo analista, encontram-se na Tabela 3 e demonstram a repetibilidade dos resultados, analisados por área como por altura, tendo valores de coeficiente de variação menores que $0,5 \%$.

Os resultados das injeções de uma mesma concentração realizadas em dias diferentes pelo mesmo analista ou por analistas diferentes encontram-se na Tabela 4. A análise de variância demonstra não haver diferença significativa ao nível de $95 \%$ de confiança entre todos os pares de valores.

Foi constatado que o método é bastante sensível a modificações da forma de preparação da fase móvel, como ilustram os dados da Tabela 5, referentes aos dados de área e altura para o pico do lapachol, 
Tabela 3. Avaliação da repetibilidade do lapachol em relação à área e altura

\begin{tabular}{lcc}
\hline & Área & Altura $(\mathrm{cm})$ \\
\hline Dados & $10.694 .480 \mathrm{VB}$ & 1348.608 \\
& $10.700 .296 \mathrm{~PB}$ & 1341.440 \\
& $10.696 .592 \mathrm{~PB}$ & 1353.216 \\
& $10.694 .544 \mathrm{~PB}$ & 1350.656 \\
& $10.674 .776 \mathrm{~PB}$ & 1345.024 \\
Média & $10.689 .632 \mathrm{~PB}$ & 1348.096 \\
Desvio padrão & 10.691 .720 & 1347.840 \\
Coeficiente de Variação (\%) & 8992.58 & 4.156 \\
\hline
\end{tabular}

Tabela 4. Avaliação da reprodutibilidade do lapachol

\begin{tabular}{lccc}
\hline $\begin{array}{l}\text { Analista } \\
\text { Dia }\end{array}$ & 1 & 2 & A \\
\hline Média & $5.486 .534,5$ & $5.340 .423,25$ & $5.416 .555,8$ \\
Variância & $1,50752 \times 10^{10}$ & $4,92164 \times 10^{9}$ & $4,88665 \times 10^{9}$ \\
Desvio padrão & $122.781,1$ & $70.154,4$ & $69.904,6$ \\
Coeficiente de variação & $2,2379 \%$ & $1,3136 \%$ & $1,2906 \%$ \\
\hline
\end{tabular}

Tabela 5. Avaliação da robustez do método de dosagem do lapachol

\begin{tabular}{lcccccc}
\hline & \multicolumn{2}{c}{ Balão } & \multicolumn{2}{c}{ Proveta } & \multicolumn{2}{c}{ CLAE } \\
& Área & Altura $(*)$ & Área & Altura $(*)$ & Área & Altura $(*)$ \\
\hline 1143949 & 144,704 & 1060262 & 129,408 & 1134899 & 144,320 \\
& 1144094 & 144,800 & 1068458 & 130,080 & 1112294 & 144,032 \\
& 1152584 & 145,088 & 1071394 & 129,088 & 1132881 & 145,280 \\
& 1146778 & 145,088 & 1062848 & 129,728 & 1152405 & 144,929 \\
Média & 1144738 & 144,736 & 1077589 & 130,016 & 1134703 & 145,344 \\
D.P. & 1148348 & 144,512 & 1062131 & 129,984 & 1136145 & 145,088 \\
C.V. $(\%)$ & 1146749 & 144,821 & 1067114 & 129,717 & 1133888 & 144,832 \\
& 3332,8 & 0,2278 & 6630,7 & 0,3959 & 12782,6 & 0,5366 \\
\hline As & 0,2498 & 0,1573 & 0,6214 & 0,3052 & 1,1273 & 0,3705 \\
\hline
\end{tabular}

(*) As medidas da altura, em cm, correspondem a medida equivalente à obtida em atenuação 0 (zero), segundo especificações técnicas do fabricante do integrador HP

obtidos com fases móveis preparadas em proveta ou em balão volumétrico, ou ainda misturados pelo próprio equipamento durante a análise. Com os dados desta tabela, foi realizada a análise de variância entre pares de colunas. Apenas os valores de altura obtidos com fase móvel preparada em balão e os obtidos com a mistura diretamente em CLAE não são significativamente diferentes ao nível de $95 \%$ de confiança.

Todos os demais pares de valores foram considerados significativamente diferentes. Assim como o grau de dispersão das médias, representado pelo respectivo desvio padrão e ainda pelo coeficiente de variação, para os valores obtidos com a fase móvel preparada em balão volumétrico apresenta-se menor, optou-se por utilizá-lo em todas as demais análises.

Através da medida da linearidade, com os dados obtidos por área, foi calculado o limite de detecção e quantificação através das Equações 1 e 2, respectivamente, tendo sido obtidos os valores $\quad 0,318$ $\mu \mathrm{g} / \mathrm{mL}$ para o limite de detecção e de $0,953 \mu \mathrm{g} / \mathrm{mL}$ para o limite de quantificação. Esses resultados demosnstram que o método proposto é suficientemente sensível para detectar e quantificar uma amostra na qual cada $20 \mu \mathrm{L}$ contenha $6,4 \mathrm{ng}$ e 19,0 ng de lapachol e $\beta$-lapachona respectivamente. Esses valores são menores que os citados por Awang ${ }^{5}$, que apresenta 100 ng como o limite de detecção para o lapachol a 254 $\mathrm{nm}$. O próprio ensaio de linearidade demonstra ser capaz de quantificar concentrações de $0,05 \mu \mathrm{g} / \mathrm{mL}$, correspondendo a uma quantidade de $2,5 \mathrm{ng}$ de lapachol num volume injetado de $20 \mu \mathrm{L}$, portanto inferior àquele determinado pelas equações abaixo:

$L D=3,33 \frac{D P}{B}$

$L Q=10 \frac{D P}{B}$
LD e LQ, Limite de detecção e de quantificação, respectivamente; DP é o desvio padrão da reta de calibração;

B é o coeficiente angular da reta de calibração

\section{CONCLUSÃO}

O método analítico proposto para a detecção e quantificação do lapachol por CLAE, usando coluna Lichrosorb RP-18 250x4 mm e fase móvel constituída por metanol: solução aquosa de ácido acético a 5\% (80:20) preparada em balão volumétrico, detecção em UV a $278 \mathrm{~nm}$, mostrou-se sensível, preciso, econômico, reprodutível, robusto e linear nas faixas de 0,05 a $100 \mu \mathrm{g} / \mathrm{mL}$, sendo adequado à avaliação da qualidade de amostras diversas que contenham lapachol.

\section{REFERÊNCIAS}

1. Rao, R. V.; McBride, T. J.; Oleson, J. J.; Cancer Res. 1968, 28, 1952.

2. Fonseca, S. G. C.; Tese de Mestrado, Universidade Federal de Pernambuco, Brasil, 2001.

3. The Index Merck, 12 ${ }^{\mathrm{a}}$ ed., 1996.

4. European Agency for the Evaluation of Medicinal Products: ICH topic Q$2 b$, London. 1996,

4. ICH topic $Q-2 b$ Validation of analytical procedures: methodology, European Agency for the Evaluation of Medicinal Products, London, 1996.

5. Awang, D. V. C.; Kindack, D.; Dawson, B. A.: J. Chromatogr. 1986, 368, 439.

6. Glen, V. L.; Hutson, P. P.; Kehrli, N. J.; Bootman, D. A.; Wilding, G.; J. Chromatogr., B: Anal. Technol. Biomed. Life Sci. 1997, 692, 181.

7. Fonseca, S. G. C.; Castro, R. F.; Santana, D. P.; Resumos do 6o Pharmatech, Recife, Brasil, 2001. 